\title{
GPS/GLONASS Carrier Phase Elevation-Dependent Stochastic Modelling Estimation and Its Application in Bridge Monitoring
}

\author{
Ruijie Xi ${ }^{1,2}$, Xiaolin Meng ${ }^{2}$, Weiping Jiang*1,3, Xiangdong An ${ }^{2,3}$, Qusen Chen ${ }^{1,2}$ \\ ${ }^{1}$ School of Geodesy and Geomatics, Wuhan University, 129 Luoyu Road, Wuhan 430079, Hubei, China; \\ ${ }^{2}$ Nottingham Geospatial Institute, The University of Nottingham, Nottingham NG7 2TU, United Kingdom; \\ ${ }^{3}$ GNSS Research Center, Wuhan University, 129 Luoyu Road, Wuhan 430079, Hubei, China.
}

\begin{abstract}
The Global Positioning System (GPS) based monitoring technology has been recognised as an essential tool in the long-span bridge health monitoring throughout the world in recent years. However, the high observation noise is still a big problem that limits the high precision displacement extraction and vibration response detection. To solve this problem, GPS double-difference model and many other specific function models have been developed to eliminate systematic errors e.g. unmodeled atmospheric delays, multipath effect and hardware delays. However, relatively less attention has been given to the noise reduction in the deformation monitoring area. In this paper, we first proposed a new carrier phase elevation-dependent precision estimation method with GeometryFree (GF) and Melbourne-Wübbena (MW) linear combinations, which is appropriate to regardless of Code Division Multiple Access (CDMA) system (GPS) or Frequency Division Multiple Access (FDMA) system (GLONASS). Then, the method is used to estimate the receiver internal noise and the realistic GNSS stochastic model with a group of zero-baselines and short-baselines (served for the GNSS and Earth Observation for Structural Health Monitoring of Bridges (GeoSHM) project), and to demonstrate their impacts on the positioning. At last, the contribution of integration of GPS and GLONASS is introduced to see the performance of noise reduction with multi-GNSS. The results show that the higher level receiver internal noise in cost effective receivers has less influences on the short-baseline data processing. The high noise effects introduced by the low elevation satellite and the geometry variation caused by rising and dropping satellites, can be reduced by $10 \%-20 \%$ with the refined carrier phase elevation-dependent stochastic model. Furthermore, based on observations from GPS and GLONASS with the refined stochastic model, the noise can be reduced by $30 \%-40 \%$, and the spurious signals in the real-life bridge displacements tend to be completely eliminated.
\end{abstract}


Keywords: GPS/GLONASS; Bridge deformation monitoring; Carrier phase elevation-dependent stochastic model; Noise reduction; Displacement; Vibration response extraction.

\section{Introduction}

Global Navigation Satellite System (GNSS) is now gradually recognized as an essential tool for Structural Health Monitoring (SHM) of bridges, high-rise buildings, dams and other civil engineering infrastructures. In recent years, a large number of case studies on deformation monitoring of large bridges using GNSS have been carried out (Meng et al., 2003, Meng et al., 2004, Chan et al. 2006, Meng 2007, Psimoulis et al., 2008, Moschas and Stiros 2011, Yi et al., 2013, Yu et al., 2014, Han et al., 2016), and its ability of providing 10-20 mm precision displacement and of identifying up to $10 \mathrm{~Hz}$ frequency vibration signal has been acknowledged. The natural frequencies of the bridge could even be detected with the spectral content of the signal-to-noise (SNR) of GPS signals (Peppa et al., 2018). The latest advancements on GNSS based SHM method and platform development can be found from Yu et al. (2016), Jiang et al. (2017), Xiong et al. (2017), Roberts et al. (2017), Peppa et al. (2018) and Meng et al. (2018).

Generally, for bridge deformation monitoring, Real-time Kinematic (RTK) GNSS data processing is always applied to provide instantaneous three-dimensional (3D) positions of monitoring points (Tamura et al., 2002, Guo et al., 2005, Elnabwy et al., 2013, Górski 2017). For attaining high precision, one or more reference station(s) will be established near the bridge at a(the) stable place(s) to form short baselines $(<5 \mathrm{~km})$ with monitoring stations. Due to the short baseline, double-difference (DD) technology is widely used to eliminate the common errors in both stations' GNSS measurements. To further improve the efficiency of positioning, the baseline length constraint, monitoring station coordinate constraint and triple difference methods were proposed. All of them have been confirmed a good performance in the real-life bridge monitoring experiments (Dai et al., 2007, Liu et al., 2011).

However, the large background noise commonly exists in the GNSS observation. Hence, the low-frequency component (primarily under $0.05 \mathrm{~Hz}$ ) of the displacement time series will contain bias, and, in the high-frequency component, the vibration amplitude of the response under the ambient excitation will contain false distortion signal or be covered by noise (Górski 2017). This is still a big problem that prevents the GNSS based monitoring technology to extract displacements in 
high accuracy and to detect correct vibration response. However, the noise level control method in deformation monitoring is still rarely studied.

The GNSS observation noise mainly comes from receiver internal noise, multipath effects and residual atmosphere delays, etc. (Amiri-Simkooei and Tiberius, 2007, Amiri-Simkooei 2009). Apart from the functional model of the GNSS data processing, a stochastic model describes the observation precision relating receiver internal noise and residual errors, and the correlations between the variables ( $\mathrm{Li}$ et al., 2015a, Li 2016, Li et al., 2017a). The adequate stochastic model is a precondition for the reliable ambiguity resolution and precise positioning (Teunissen 2007, AmiriSimkooei et al., 2016). Hence, significant research efforts have been put in refining the GNSS stochastic model, and the elevation dependence characteristic of GNSS, the cross-correlation between observation types and time-correlation have been acknowledged (Amiri-Simkooei et al., 2013a, Amiri-Simkooei 2013b, Li et al., 2015b). However, the empirical elevation-dependent models are still widely used in the GPS data processing software, for instance, GAMIT and Bernese (Dach et al., 2007, Herring et al., 2010). This may result in a lower integer ambiguity resolution successful rate and positioning accuracy (Li et al., 2008, Yang et al., 2017, Schön and Brunner 2008). In this case, for the high accuracy monitoring of displacements, the realistic stochastic model estimation and application could be a way to deal with the high level noise in SHM and needs to be further studied. In addition, much efforts have been made in positioning with data from multiple GNSS (Multi-GNSS). Its great advantages include the increasing of the number of visible satellites, the strong geometric strength, the rapid ambiguity resolution and the high positioning precision (Odolinski et al., 2015, Teunissen et al., 2014, Yu et al., 2017, Li et al., 2017b, Liu et al., 2017, Geng et al., 2017). As a consequence, multi-GNSS could be an another way to reduce the noise level and eliminate the false distortion signals. However, the methods often used are only appropriate to CDMS system, such as GPS and BeiDou Navigation Satellite System (BDS).

In this study, we firstly proposed a new single differential (SD) geometry-free method to estimate the carrier phase elevation-dependent precisions of GPS and GLONASS with MW and GF combinations. Then, based on a testing platform served for the GeoSHM project, a project sponsored by the European Space Agency, the internal noise of two receiver brands and a realistic stochastic elevation based model was built with a group of zero baselines and short baselines. Thirdly, the effect of the realistic GNSS elevation-dependent weighting and integration of GPS and GLONASS 
on positioning are numerically analysed. Finally, a real-life bridge monitoring data set is applied to demonstrate the performance of the realistic GNSS elevation-dependent stochastic model and the multi-GNSS application in the false distortion signal elimination.

\section{Elevation-dependent precision estimation with SD geometry-free method}

\subsection{The SD Geometry-free functional model}

As pseudorange is easily contaminated by multipath and hardware delays, biases in the pseudorange are significant (Yu et al., 2017, Chu and Yang 2018, Chu et al., 2016). Hence, only carrier phase observations are usually used in our monitoring work and only the elevation-dependent precision of carrier phase is estimated in this study. As proposed by Li et al. (2016), SD geometryfree stochastic model estimation has the advantages of only one satellite involving in a SD observation and no mathematical correlation between satellites, which is suitable for the stochastic modelling estimation. On ultrashort (shorter than $10 \mathrm{~m}$ ) or zero baselines, the systematic errors can be assumed to be completely eliminated in a SD observation. In this way, only the pure random errors remain, which can help us to estimate the precision of the satellite-specific variances (the stochastic model).

Then the single-epoch, single-frequency between-receiver SD geometry-free GPS or GLONASS phase observation equation can be read

$$
\lambda_{j} \Delta \phi_{j}=\Delta \rho+e_{s} \Delta t_{j}+\lambda_{j} \Delta N_{j}+\Delta \varepsilon
$$

where the subscripts $j$ is the frequency number $(j=1,2) ; s$ is the number of tracked GPS or GLONASS satellites, $\Delta \phi_{j}=\left[\Delta \phi_{1, j}, \cdots, \Delta \phi_{s, j}\right]^{T}$ is the vectors of the $j$-frequency SD phase observations expressed in cycles; $\Delta \rho=\left[\Delta \rho^{1}, \cdots, \Delta \rho^{s}\right]^{T}$ denotes the vector of $s$ SD satellitereceiver ranges; $e_{s}$ denotes the $s$-column vector with all elements of ones; $\Delta t_{j}$ is the SD receiver clock errors for phase measurements and also including hardware delays; $\lambda_{j}$ is the wavelength of the $j$-th frequency; $\Delta N_{j}=\left[\Delta N_{1, j}, \cdots, \Delta N_{s, j}\right]^{T}$ is the SD integer ambiguity vector of the $j$-th frequency, which are expressed in cycles. Note that in this study, the receivers we use are with the 
same hardware configuration (i.e., same manufacturer, receiver type, firmware version, and antenna type) for GLONASS data. Thus, the inter-frequency bias (IFB) will be out of the consideration [44]. In this study, the zero baseline and ultrashort baseline are used, and the antennas are set on the known positions. In theory, $\Delta \rho$ equal zeros in the zero-baseline or obtained by the known shortbaseline, which can be moved to the left side of the observation equations. Then, the parameters $\Delta t_{j}$ and $\Delta N_{j}$ are left. However, Eq. (1) is rank-deficient since the coefficients of $\Delta t_{j}$ and $\Delta N_{j}$ satisfy

$$
\left[e_{s}, \lambda_{j} I_{s}\right]\left[\begin{array}{c}
\lambda_{j} \\
-e_{s}
\end{array}\right]=0_{s \times 1}
$$

That means $\Delta t_{j}$ are dependent on the SD ambiguities $\Delta N_{j}$ with rank deficiency 1. In this case, we propose a new method to obtain the fixed SD ambiguities $\Delta N_{j}$ in Eq. (1) directly for every satellite without reference satellite.

Geometry-Free (GF) and Melbourne-Wübbena (MW) linear combination are two combinations often used in the cycle slip detection and the ambiguity resolution, due to the advantages of eliminating the satellite clock offset, the receiver clock error and the distancedependent term (such as tropospheric and ionospheric delay). The single difference MW and GF combinations with the dual-frequency carrier phase are:

$$
\left\{\begin{array}{l}
\Delta \phi_{M W}=\Delta \phi_{1}-\Delta \phi_{2}-\frac{1-g}{1+g}\left(\frac{\Delta P_{1}}{\lambda_{1}}+\frac{\Delta P_{2}}{\lambda_{2}}\right)=\Delta N_{2}-\Delta N_{1} \\
\Delta \phi_{G F}=\Delta \phi_{1}-\frac{1}{g} \Delta \phi_{2}=\frac{1}{g} \Delta N_{2}-\Delta N_{1}
\end{array}\right.
$$

where $g=f_{2} / f_{1} \cdot f_{1}$ and $f_{2}$ are frequencies of GPS or GLONASS dual-frequency observation; $\Delta P_{1}$ and $\Delta P_{2}$ are $\mathrm{SD}$ pseudorange observations, and $\lambda_{1}, \lambda_{2}$ are the corresponding wavelengths. The standard deviation of MW and GF combinations are

$$
\left\{\begin{array}{l}
\sigma_{\Delta \phi_{M W}}=\sqrt{2} \sqrt{\sigma_{\phi_{1}}^{2}+\sigma_{\phi_{2}}^{2}+\left(\frac{1-g}{1+g}\right)^{2} \sqsubset \sigma_{P_{1}}^{2}+\left(\frac{1-g}{1+g}\right)^{2} \sqsubset \sigma_{P_{2}}^{2}} \\
\sigma_{\Delta \phi_{G F}}=\sqrt{2} \sqrt{\sigma_{\phi_{1}}^{2}+\frac{1}{g^{2}} \llbracket \sigma_{\phi_{2}}^{2}}
\end{array}\right.
$$


where $\sigma_{\phi}$ and $\sigma_{P}$ are the undifferenced standard deviation in theory.

Based on Eq. (3), the float solution of SD ambiguities in the two frequencies can be solved as:

$$
\left\{\begin{array}{l}
\Delta \hat{N}_{1}=\frac{1}{g-1} \Delta \phi_{M W}+\frac{g}{g-1} \Delta \phi_{G F} \\
\Delta \hat{N}_{2}=\frac{g}{g-1} \Delta \phi_{M W}+\frac{g}{g-1} \Delta \phi_{G F}
\end{array}\right.
$$

Table 1 GPS and GLONASS dual-frequency signals. ( $\mathrm{k}$ is a frequency number of the signals transmitted by GLONASS satellites.)

\begin{tabular}{ccc}
\hline Sat. System & L1 $(\mathrm{MHz})$ & $\mathrm{L} 2(\mathrm{MHz})$ \\
\hline GPS & $1,575.42$ & $1,227.60$ \\
GLONASS & $1,602+\mathrm{k} * 0.5625$ & $1,246+\mathrm{k} * 0.4375$ \\
\hline
\end{tabular}

According to the frequencies of GPS and GLONASS dual-frequency signals in Table 1, Eq. (5) can be rewritten as

$$
\begin{aligned}
& \left\{\begin{array}{l}
\Delta \hat{N}_{1}=\frac{77}{17} \Delta \phi_{M W}-\frac{60}{17} \Delta \phi_{G F} \\
\Delta \hat{N}_{2}=\frac{60}{17} \Delta \phi_{M W}-\frac{60}{17} \Delta \phi_{G F} \quad \text { for } \quad G P S
\end{array}\right. \\
& \left\{\begin{array}{l}
\Delta \hat{N}_{1}=\frac{9}{2} \Delta \phi_{M W}-\frac{7}{2} \Delta \phi_{G F} \quad \text { for } \quad \text { GLONASS } \\
\Delta \hat{N}_{2}=\frac{7}{2} \Delta \phi_{M W}-\frac{7}{2} \Delta \phi_{G F}
\end{array}\right.
\end{aligned}
$$

and the accuracy of SD ambiguities are

$$
\begin{aligned}
& \left\{\begin{array}{l}
\sigma_{\Delta \hat{N}_{1}}=\sqrt{\left[\left(\frac{77}{17}\right)^{2} \sigma_{\Delta \phi_{M W}}^{2}+\left(\frac{60}{17}\right)^{2} \sigma_{\Delta \phi_{G F}}^{2}\right]} \\
\sigma_{\Delta \hat{N}_{2}}=\sqrt{\left[\left(\frac{60}{17}\right)^{2} \sigma_{\Delta \phi_{M W}}^{2}+\left(\frac{60}{17}\right)^{2} \sigma_{\Delta \phi_{G F}}^{2}\right]}
\end{array} \text { for } G P S\right. \\
& \left\{\begin{array}{l}
\sigma_{\Delta \hat{N}_{1}}=\sqrt{\left[\left(\frac{9}{2}\right)^{2} \sigma_{\Delta \phi_{M W}}^{2}+\left(\frac{7}{2}\right)^{2} \sigma_{\Delta \phi_{G F}}^{2}\right]} \\
\sigma_{\Delta \hat{N}_{2}}=\sqrt{\left[\left(\frac{7}{2}\right)^{2} \sigma_{\Delta \phi_{M W}}^{2}+\left(\frac{7}{2}\right)^{2} \sigma_{\Delta \phi_{G F}}^{2}\right]}
\end{array}\right. \text { for GLONASS }
\end{aligned}
$$


With Eq. (4) and Eq. (7), the accuracy of estimated SD ambiguities can be obtained. To fixed the ambiguities estimated in Eq. (6), in this study, a small search produce is applied and the search space is decided by the accuracy of the float SD ambiguities, as

$$
\left\{\begin{array}{l}
\Delta \bar{N}_{1} \in\left[\operatorname{round}\left(\Delta \hat{N}_{1}\right)-5\left\lceil\sigma_{\Delta \hat{N}_{1}}\right\rceil, \operatorname{round}\left(\Delta \hat{N}_{1}\right)+5\left\lceil\sigma_{\Delta \hat{N}_{1}}\right\rceil\right] \\
\Delta \bar{N}_{2} \in\left[\operatorname{round}\left(\Delta \hat{N}_{2}\right)-5\left\lceil\sigma_{\Delta \hat{N}_{2}}\right\rceil, \operatorname{round}\left(\Delta \hat{N}_{2}\right)+5\left\lceil\sigma_{\Delta \hat{N}_{2}}\right\rceil\right]
\end{array}\right.
$$

where $\Delta \bar{N}_{1}$ and $\Delta \bar{N}_{2}$ is the candidates and " \lceil\rceil " means rounding up to the nearest integer. "round" indicates fixing to its nearest integer. Generally, without significant disturbed delays and multipath effects, we assume the standard deviation of undifferenced carrier phase and code measurements are $0.5 \mathrm{~cm}$ and $1 \mathrm{~m} .5\left[\sigma_{\Delta \hat{N}_{1}}\right\rceil$ and $5\left\lceil\sigma_{\Delta \hat{N}_{2}}\right\rceil$ will be limited into only 2 cycles. Then, $\Delta \breve{N}_{1}$ and $\Delta \breve{N}_{2}$ are fixed ambiguities with the candidates $\Delta \bar{N}_{1}$ and $\Delta \bar{N}_{2}$ who satisfy

$$
\left\{\begin{array}{l}
r_{M W}=\left[\Delta \phi_{M W}-\left(\Delta \bar{N}_{1}-\Delta \bar{N}_{2}\right)\right]^{2} \\
r_{G F}=\left[\Delta \phi_{G F}-\left(\Delta \bar{N}_{1}-\frac{1}{g} \Delta \bar{N}_{2}\right)\right]^{2} \\
\left(r_{M W}+r_{G F}\right)=\min
\end{array}\right.
$$

After fixing the SD ambiguities, the Eq. (1) can be rewritten as

$$
E\left(\overline{\lambda_{j} \Delta \phi_{j}}\right)=e_{s} \Delta t_{j}
$$

where

$$
\overline{\lambda_{j} \Delta \phi_{j}}=\lambda_{j} \Delta \phi_{j}-\Delta \rho-\lambda_{j} \Delta \breve{N}_{j}=l_{j}
$$

and only $\Delta t_{j}$ left to be estimated. To resolve parameters, the least squares model was applied, read as

$$
y=B x+\varepsilon_{y}, \quad Q_{y y}=\operatorname{blkdiag}\left(Q_{\phi \phi}\right)
$$

where $y=\left[l_{1, j}, \cdots, l_{s, j}\right]^{T} ; \quad B=I_{2} \otimes e_{s}$ is the design matrix; and $x=\left[\Delta t_{j}\right]^{T}$. The symbols $\otimes$ is Kronecker product. $\quad \varepsilon_{y}$ is the random observation noise assumed to be normally distributed with zero mean and covariance matrix of $Q_{y y} . Q_{y y}$ is the covariance matrices of SD phase observations. 
The benefit of this method is that it not only can be used in CDMA systems, including GPS, BeiDou and Galileo, but also available for FDMA system (GLONASS).

\subsection{Carrier Phase Elevation-Dependent Precision Estimation}

In this study, we only consider the effect of elevation-dependent precision on structural monitoring, and the cross and time correlation within observations are ignored, due to they have a marginal effect on positioning solutions (Li 2016).

In general, the variance of satellites' observations is constant for a slight elevation angle variation. Meanwhile, in order to smooth the noise in MW observations mainly caused by pseudorange measurements to improve the reliability of SD ambiguity fixing, $n$ consecutive epochs over a short time duration are resolved together. The observation equation of $n$ epochs are read as

$$
\bar{y}=\left(I_{n} \otimes B\right) \bar{x}+\bar{\varepsilon}_{y}
$$

where $\bar{y}=\left[y_{1}, \cdots, y_{n}\right]^{T} ; \quad \bar{x}=\left[x_{1}, \cdots, x_{n}\right]^{T}$. Since the variance is unique over these $n$ epochs for one satellite, the stochastic model for Eq. (13) can be written as

$$
Q_{\overline{y y}}=I_{n} \otimes Q_{\Delta \phi_{j}}
$$

where

$$
Q_{\Delta \phi_{j}}=2 \times \operatorname{diag}\left(\left[\sigma_{1, j}^{2}, \cdots, \sigma_{s, j}^{2}\right]\right)
$$

is the variance matrix of SD phase observation on $j$-frequency, and $\sigma_{i, j}$ is the precision of undifferenced observation for the $i$-th satellite. It can be estimated by Eq. (16) (Li et al., 2015a)

$$
\hat{\sigma}_{i, j}=\sqrt{\frac{\sum_{k=1}^{n}\left(\breve{\varepsilon}_{y_{i, j}}^{k}\right)^{2}}{2 n r_{i, j}}}
$$

where $\breve{\varepsilon}_{y_{i, j}}^{k}$ is the residual SD residuals of the $j$-frequency of the $i$-th satellite at $k$ epoch. $r_{i, j}$ is the redundancy of the $i$-th satellite. It can be calculated by

$$
r_{i, j}=(s-1) / s
$$

For more details regarding derivation of Eq. (16) and (17), please see Li et al. (2015a). 
Finally, the precisions of phase observations for the $i$-th satellite associate with the mean value of elevation angles over $n$ epochs can be obtained by Eq. (16). In this paper, we estimate the precision of phase observation every $1^{\circ}$ elevation angle.

\section{Experiments and result analysis}

\subsection{Data description}

GeoSHM (GNSS and Earth Observation for Structural Health Monitoring of Bridges) is a feasibility study project funded under the Integrated Application Promotion (IAP) program of the European Space Agency (ESA) in August 2013 (Meng et al., 2018). It is a system that uses integrated GNSS, Earth Observation technologies and environmental data to offer bridge owners an effective tool to assess the operational conditions of their assets. In order to make a system that is more robust, accurate, useful, reliable and cost-effective, a receiver assessing and data quality testing platform served for GeoSHM with zero-baselines and short-baselines was established on the roof of the Nottingham Geospatial Building on the Jubilee Campus of the University of Nottingham, UK.

A GNSS reference station (named SHM7) with a Leica GM30 receiver and a LEIAR 10 antenna was set on a permanent point and four receivers with two LEICA GM30 receivers (named SHM5 and SHM6) and two PANDA TI4100 receivers (named SHM8 and SHM9), developed by Wuhan University in China, were connected via a signal splitter (GPS RMS18 splitter) with a LEIAR 10 antenna on an experiment point (monitoring station). In this case, the monitoring station with four receivers is the zero baseline. The short baseline is formed by the reference and monitoring stations, and linked to the baseline length $6.97 \mathrm{~m}$. Both antennas were mounted in an open sky environment. Fig 1 illustrates the configuration of the baselines includes the receivers and the splitter used in this experiment.

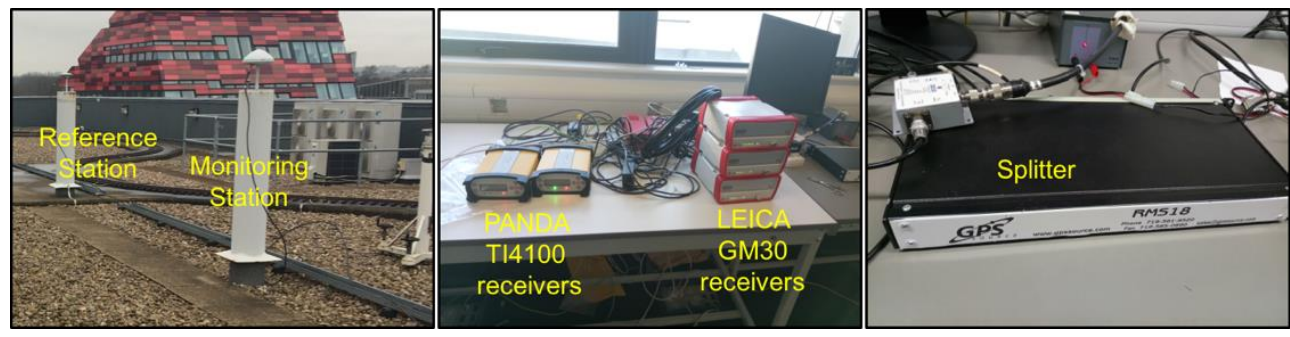

Fig 1 Zero and short baseline experiment setup, the receivers and the splitter. 
All the LEICA receivers were set to collect GPS and GLONASS data, and the PANDA receivers collect only GPS data. The data were collected for two days (48 hour) with a sampling interval of $1 \mathrm{~s}$. The cutoff elevation was set to $10^{\circ}$. Fig 2 shows the sky plot of the GPS and GLONASS visibility and the number of satellites observed with its corresponding Position Dilution of Precision (PDOP) values during the whole day. In the experimental session, 31 GPS satellites were tracked without G04 and 23 GLONASS satellites without R12. From right panel of Fig 2, it can be seen that the number of GPS satellites observed at one epoch over the whole day is more than 8 mostly. However, the PDOP values change dramatically for GPS, due to the variation of geometry strength. For GLONASS, the visible satellite is almost less than 8 at one epoch. If assumed 8 satellites are needed to achieve reliable positioning, the GLONASS only data processing will not be shown in this paper. After integrating GPS and GLONASS data, more than 15 satellites can be observed and the PDOP value changes around 1.3. Thus, a stable precision of positioning could be shown in the GPS+GLONASS data processing.
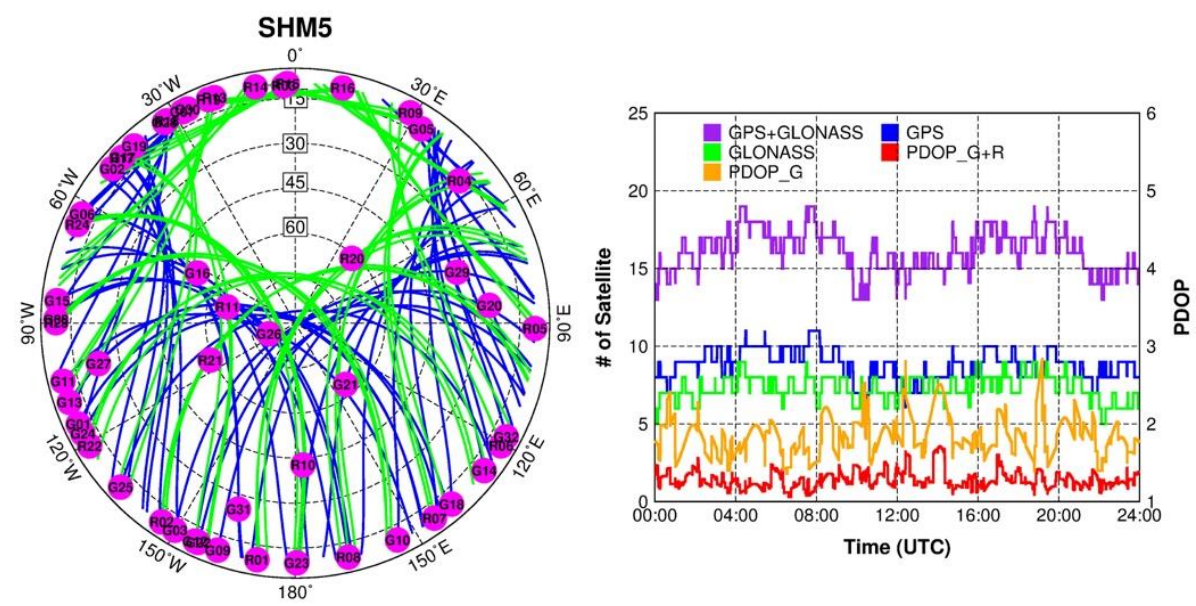

Fig 2 Sky plot and the number of visible satellites with PDOP time series for the whole day. (In the right panel, G

- GPS only; G+R - GPS+GLONASS)

\subsection{Receiver internal noise assessment}

Generally, the stochastic properties of observations are affected by both receiver-specific internal noise errors and environmental errors. At first, the zero baseline pairs SHM5-SHM6 (LEICA) and SHM8-SHM9 (PANDA) were processed respectively to assess the receiver internal noise. Based on the SD geometry-free function model, we first fixed the SD ambiguities of every satellite by MW and GF combinations with $n=300$ epochs, and then obtained the residuals of GPS and GLONASS satellites. The mean precisions of each satellite for every elevation interval of $1^{\circ}$ 
were estimated with Eq. (16). Finally, the overall precisions of all of the frequency types with elevation variation are calculated by averaging the mean precisions of all satellites. The residual time series as a function of the elevation for all of the satellites are shown in Fig 3, and the estimated precisions of L1 and L2 are shown in Fig 4. The red dash line in Fig 3 is the mean value of all of the precisions for each frequency.
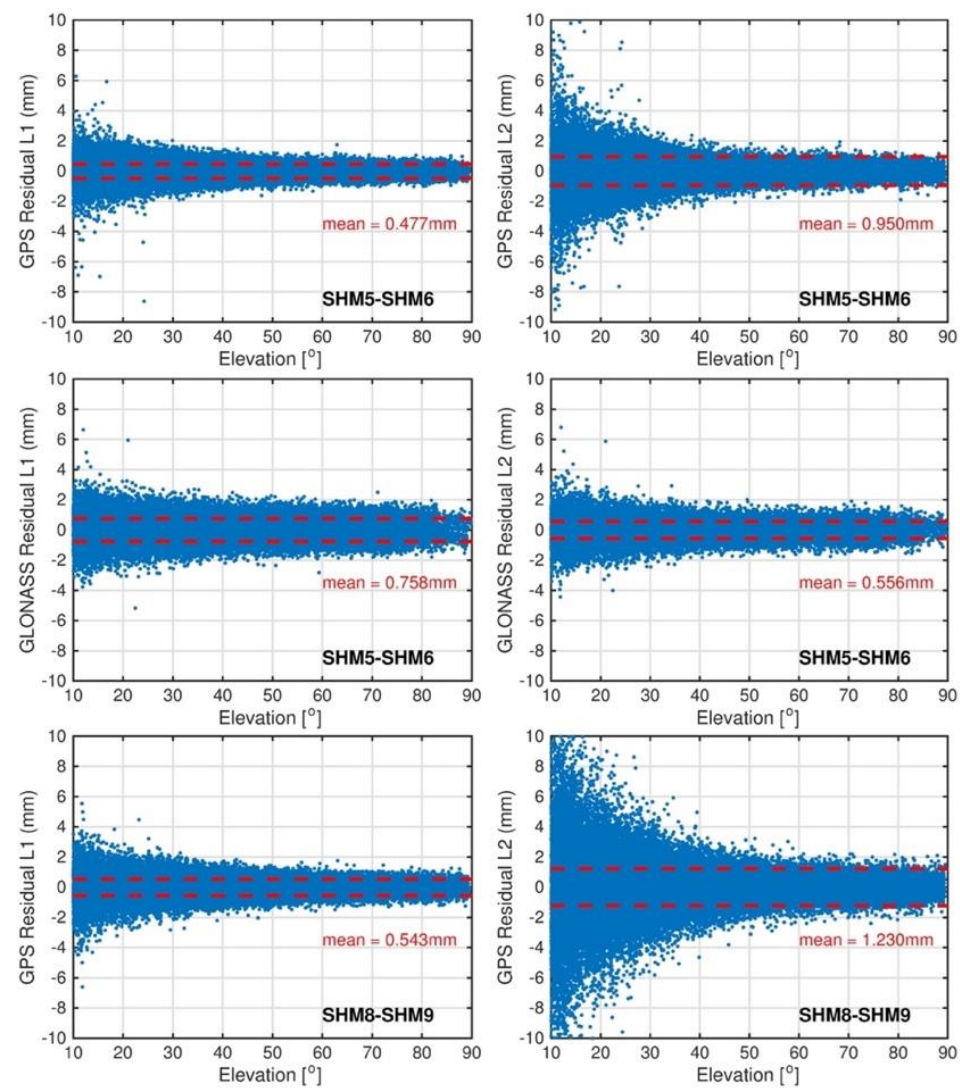

Fig 3 Residual time series of all satellites changes with elevation variation from $10^{\circ}$ to $90^{\circ}$. The red dash line is the mean precision of all satellite observation.

For Fig 3 and Fig 4, the results show that the residuals and precisions of phase observations are elevation-dependent for both systems, both frequencies and both receivers. However, different features shown in different systems, frequencies and receivers. For GPS, the residuals series of L1 are overall better than L2 in both receivers, especially in elevations lower than $40^{\circ}$. From the perspective of receivers, LEICA is slightly better than PANDA receivers on L1. However, on L2, the residuals from the PANDA receiver pairs are extremely larger than the LEICA ones. That means for two receiver pairs, the noise in L1 is smaller than L2, and the internal noise of the PANDA receiver is higher than the LEICA receiver. In contrast, for GLONASS system, the noise is lower for L2 than that of L1, and the elevation-dependent characteristic is not that obvious as GPS. 

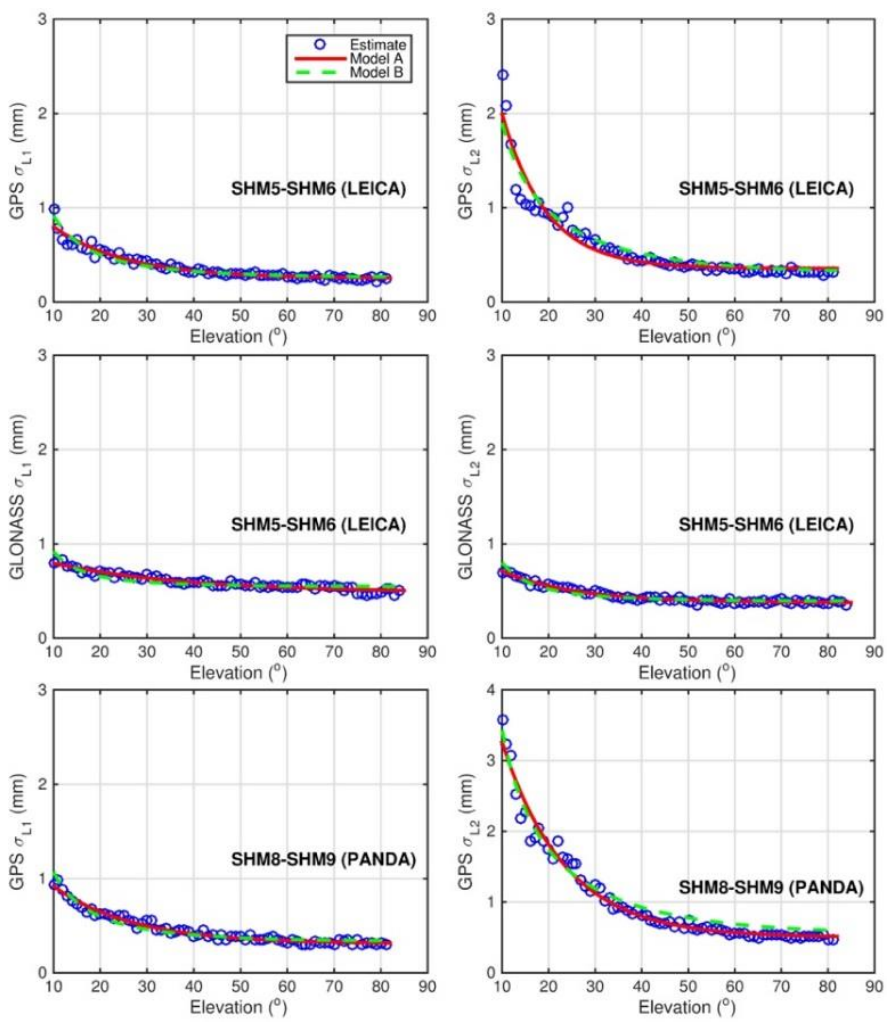

Fig 4 Estimated elevation-dependent precisions and their modeling with the two predefined models.

As proposed by Li et al. (2015a, 2016), the predefined functions of elevations, exponent type and sine type, can achieve a good result in descripting the observation precisions with elevation changes. Then, we employ the following two models to fit the precisions:

$$
\left\{\begin{array}{lc}
A: & \sigma=c_{0}+c_{1} \times \exp \left(-\theta / \theta_{0}\right) \\
B: & \sigma=\sqrt{a^{2}+b^{2} / \sin ^{2}(\theta)}
\end{array}\right.
$$

where $c_{0}, c_{1}, \theta_{0}, a$, and $b$ are the fitting parameters. Model $\mathrm{B}$ is an classic empirical model (Herring et al., 2010, Qian et al., 2016, Li and Zhang 2014) often used in the GPS data processing, and the values of $a$ and $b$ are usually set to $3 \mathrm{~mm}$. The fitted curves of Model A and Model B are shown in Fig 4, and the estimated fitting parameters can be found in Table 2.

It is clearly shown that, from Fig 4, the two models can fit the overall elevation-dependent precisions very well and they show a high agreement with each other. For L1 observations, the precision is higher than $1 \mathrm{~mm}$ for all data set. Except for GLONASS L2 observations whose precision is also better than $1 \mathrm{~mm}$, the internal noise is larger than $2 \mathrm{~mm}$ for GPS at elevation $10^{\circ}$. The lowest precision is shown in L2 frequency of the PANDA receivers with larger than $4 \mathrm{~mm}$ for $10^{\circ}$ elevation. However, the precisions tend to be constant to $0.5 \mathrm{~mm}$ for the elevation upper than $30^{\circ}$ for all observations. 
Table 2 Fitting parameters of two predefined elevation-dependent models.

\begin{tabular}{cccccccccccc}
\hline & \multicolumn{4}{c}{ L1 } & \multicolumn{1}{c}{ L2 } \\
\cline { 2 - 11 } & \multicolumn{3}{c}{ Model A } & \multicolumn{1}{c}{ Model B } & \multicolumn{3}{c}{ Model A } & Model B \\
\cline { 2 - 11 } & $c_{0}$ & $c_{1}$ & $\theta_{0}$ & $a$ & $b$ & $c_{0}$ & $c_{1}$ & $\theta_{0}$ & $a$ & $b$ \\
\hline LEICA GPS & 0.25 & 1.04 & 15.71 & 0.22 & 0.15 & 0.36 & 4.71 & 9.46 & 0.01 & 0.33 \\
LEICA GLONASS & 0.48 & 0.44 & 28.69 & 0.53 & 0.13 & 0.38 & 0.63 & 15.86 & 0.38 & 0.12 \\
PANDA GPS & 0.31 & 1.10 & 16.99 & 0.29 & 0.18 & 0.50 & 5.79 & 13.49 & 0.01 & 0.60 \\
\hline
\end{tabular}

\subsection{GPS/GLONASS carrier phase elevation-dependent precision estimation with short}

\section{baselines}

In this section, SHM7 with LEICA GM30 receiver served as the reference station and four receivers connected with a single antenna set to be a monitoring station were formed a group of short baselines. Same as zero-baselines, at first, we estimated the observation precisions based on the SD geometry-free method, and get the residuals of L1 and L2 of the four baselines for the two systems. The residual time series as a function of elevations for all satellites are shown in Fig 5.
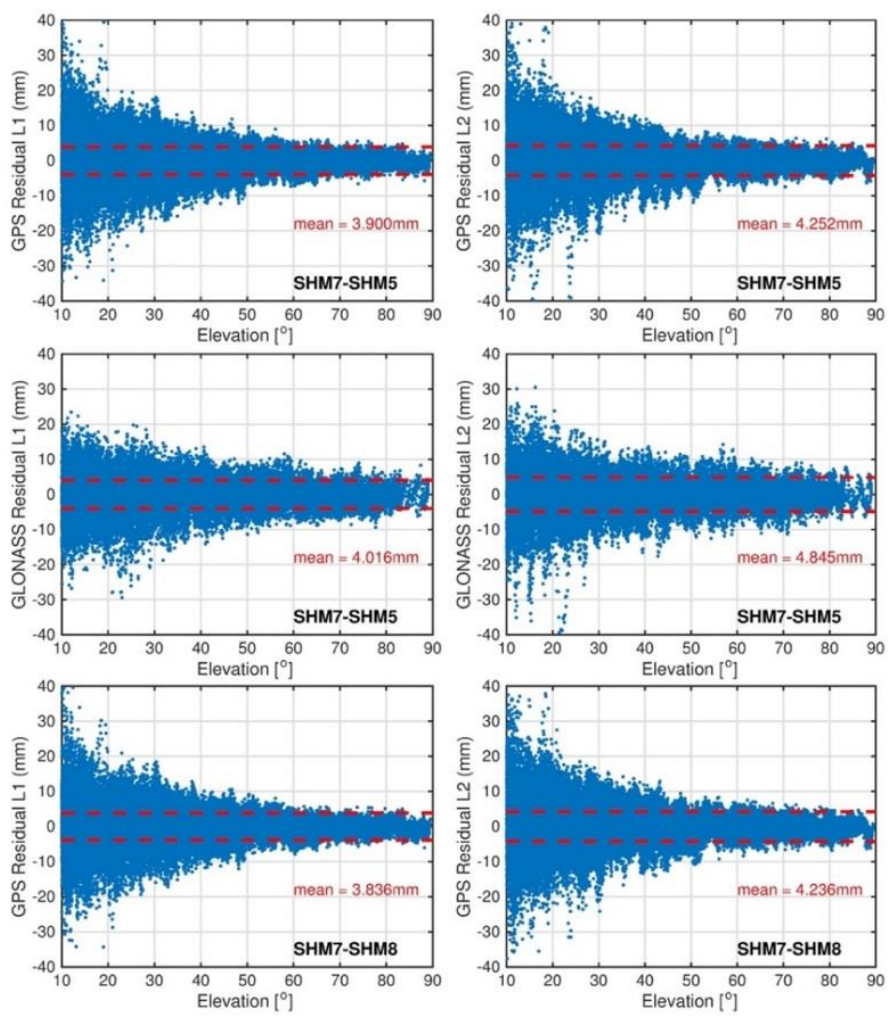

Fig 5 Residual time series changes with the elevation for all satellites. The red dash line is the mean precision of all residuals. 
As it is shown, the carrier phase residuals for all data are overall elevation-dependent. The mean precision is slightly better for L1 observations than L2. Even though the high level of internal noise, the highest precision are both found in PANDA results for the two frequencies. For the residual changes with elevation, when the elevation is lower than $30^{\circ}$, the maximum residual can even reach up to $4 \mathrm{~cm}$, mostly within $2.5 \mathrm{~cm}$ in GPS. GLONASS is slightly lower, within $2 \mathrm{~cm}$ and $2.5 \mathrm{~cm}$ for $\mathrm{L} 1$ and $\mathrm{L} 2$ observations respectively. For the elevation higher than $30^{\circ}$, the residual time series tend to converge within $4 \mathrm{~mm}$ with the increasing of elevation angles until to $90^{\circ}$. It demonstrates that the value of $3 \mathrm{~mm}$ usually used as the prior precision of GNSS measurements is unrealistic. Fig 6 gives the residual and elevation time series of PRN 1 satellite for the LEICA and the PANDA receivers in two consecutive days. We can see that the maximum residual is about 2.5 $\mathrm{cm}$ and the large residuals are shown when the elevation angles are lower than $30^{\circ}$. For the time series of two consecutive days, they match very well with each other when the time series of the second day was moved backward for 240 s to consider sidereal day effects of GPS. It can demonstrate that the precision characteristic of GNSS measurements in a specific environment is repeatable for a sidereal day. In this case, a realistic stochastic model can be established for a permanent monitoring point like this in the application of SHM to describe the precision of GNSS measurements.
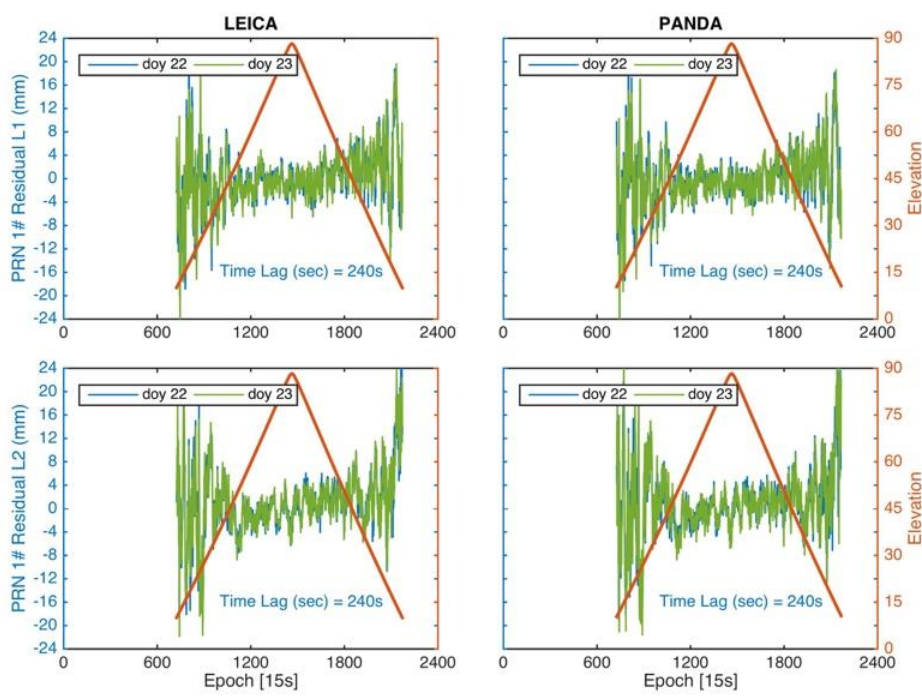

Fig 6 Residual and elevation time series of PRN 1\# in two consecutive days. The residual time series of day of year 23 was moved backward for 240 seconds (16 epochs for 15 s sampling rate)

Table 3 Fitting parameters estimated of two predefined elevation-dependent models. 


\begin{tabular}{ccccccccccc}
\hline & $c_{0}$ & $c_{1}$ & $\theta_{0}$ & $a$ & $b$ & $c_{0}$ & $c_{1}$ & $\theta_{0}$ & $a$ & $b$ \\
\hline LEICA_SHM5 GPS & 0.85 & 10.26 & 24.89 & 0.01 & 1.67 & 1.02 & 9.83 & 25.41 & 0.85 & 1.66 \\
LEICA_SHM6 GPS & 0.84 & 10.18 & 25.21 & 0.01 & 1.67 & 1.02 & 9.78 & 25.49 & 0.86 & 1.66 \\
LEICA_SHM5 GLONASS & 1.36 & 5.89 & 34.10 & 2.10 & 1.21 & 2.47 & 10.54 & 14.82 & 2.10 & 1.50 \\
LEICA_SHM6 GLONASS & 1.33 & 5.67 & 34.12 & 2.09 & 1.19 & 2.02 & 10.44 & 15.02 & 2.09 & 1.51 \\
PANDA_SHM8 GPS & 0.76 & 9.64 & 25.96 & 0.16 & 1.59 & 1.03 & 10.15 & 24.92 & 0.73 & 1.69 \\
PANDA_SHM9 GPS & 0.74 & 9.51 & 26.41 & 0.17 & 1.58 & 0.97 & 9.91 & 25.79 & 0.78 & 1.67 \\
\hline
\end{tabular}

Like what we have done for the zero-baselines, we estimated elevation-dependent precision and fitted curves with Model A and B for the short-baselines in Fig 7, and the estimated fitting parameters are shown in Table 3. It can be seen that Model A has a better fitting performance than Model B. However, the fitted curves and parameters estimated are quite similar for the two frequencies and two receiver brands. Although the receiver noise level is high for the PANDA receiver, they give almost the same result with the LEICA receiver. Based on this experiment, we know that the higher internal noise of receivers in millimeter level will not influence the baseline solution too much. The cost effective receivers can also give the same performance compared with the high quality equipment.
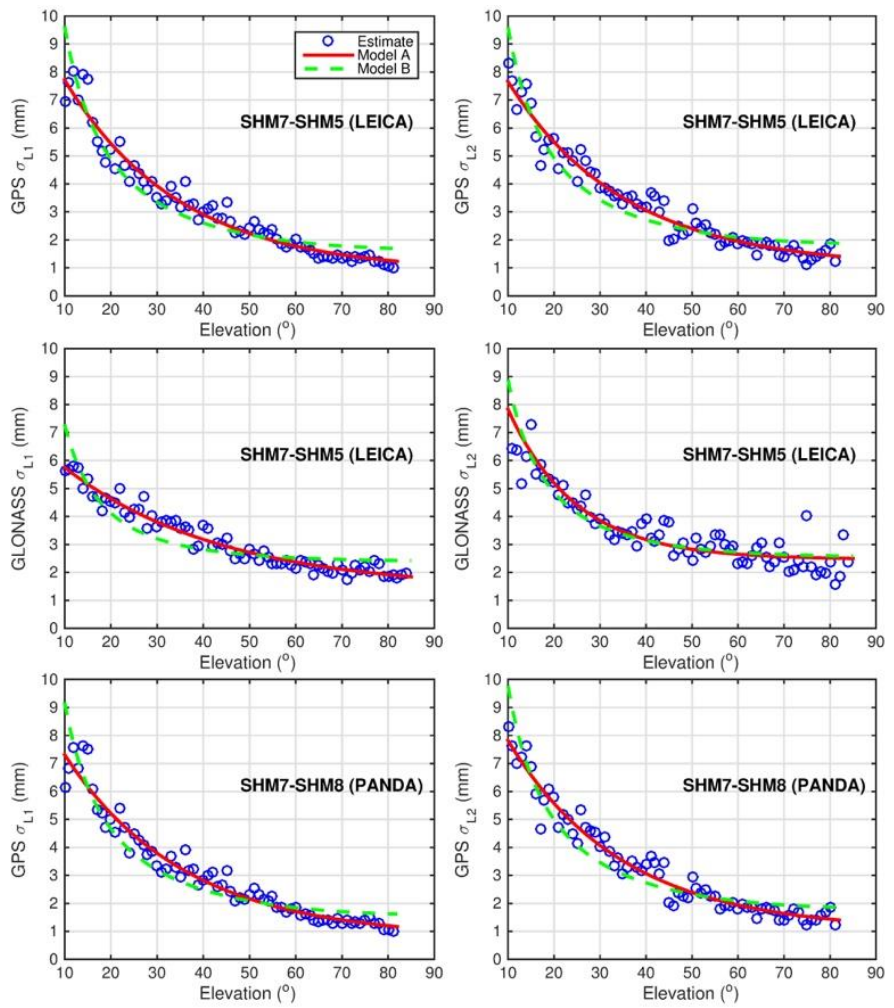

Fig 7 Estimated elevation-dependent precisions and their modelling with two predefined models in short-baselines. 
To further analyse the effect of the refined stochastic model in positioning, we processed the data of the short-baseline formed by the LEICA receiver pair and the PANDA receiver pair with the home-made GNSS real-time data processing software named GNSSDEM. The GNSSDEM software now supports dual- and triple-frequency data processing with GPS, BeiDou, GLONASS and integrated GPS, BeiDou and GLONASS using the double difference (DD) method.

Table 4 GPS and GLONASS data processing models and strategies used in GNSSDEM.

\begin{tabular}{|c|c|}
\hline Options & Processing Strategy \\
\hline Ephemeris & GPS and GLONASS broadcast ephemeris \\
\hline Signals & GPS L1/L2, GLONASS L1/L2 \\
\hline Elevation cutoff angle & $10^{\circ}$ \\
\hline Troposphere modeling & Corrected with GPT2w model (Lagler et al. 2013) \\
\hline \multirow[t]{2}{*}{ Ambiguity resolution and validation } & Wide-lane (WL) and Narrow-Lane (NL) ambiguities are solved by \\
\hline & LAMBDA with ratio $=2$ \\
\hline \multirow[t]{2}{*}{ Cycle slip detection } & SD MW+GF observations and DD ionosphere-free (IF) observations for \\
\hline & GPS and SD MW+GF observations for GLONASS \\
\hline \multirow[t]{2}{*}{ GLONASS IFB } & Out of the consideration for receivers with the same hardware \\
\hline & configurations \\
\hline
\end{tabular}

For this section, only dual-frequency data of GPS and GLONASS were processed. The data processing strategies are shown in Table 4. During the data processing, we divided the ambiguities into Wide-lane (WL) and Narrow-lane (NL) ambiguities. The WL ambiguities are resolved first with LAMBDA. Then, after WL ambiguities are fixed, the normal equation will be updated and the LAMBDA will be used to search and fix the NL ambiguities. For comparing purpose, we used the Model B with $a=b=3 m m$ (Herring et al., 2010, Qian et al., 2016, Li and Zhang 2014) as the empirical model and the Model A with parameters estimated in Table 2 as the refined model to process the GPS data only, since Model A gives a better fitting performance. The results (only SHM7-SHM5 and SHM7-SHM8) are listed in Fig 8. Comparing the refined model result with the empirical one, we can see that the precision of residual time series with the refined model seem to be improved to some extent. At the same time, the LEICA and the PANDA receiver show almost the same results. The paper also gives the standard deviations (STD) of the time series, which can be calculated with: 


$$
S T D=\sqrt{\left(\frac{1}{n-1} \sum_{i=1}^{n}\left(\Delta_{i}-\bar{\Delta}\right)^{2}\right)}
$$

where $\bar{\Delta}$ is the average bias; $n$ is the number of epochs. The statistical precision of time series are shown in Table 5. We can see that the time series with the refined model is higher than the ones with the empirical model, with the precision improvement from $10 \%$ to $20 \%$, and the four baselines show the same STD values with a same model. This also confirmed that the PANDA receiver with a higher internal noise level can have the same positioning performance with the high quality ones.
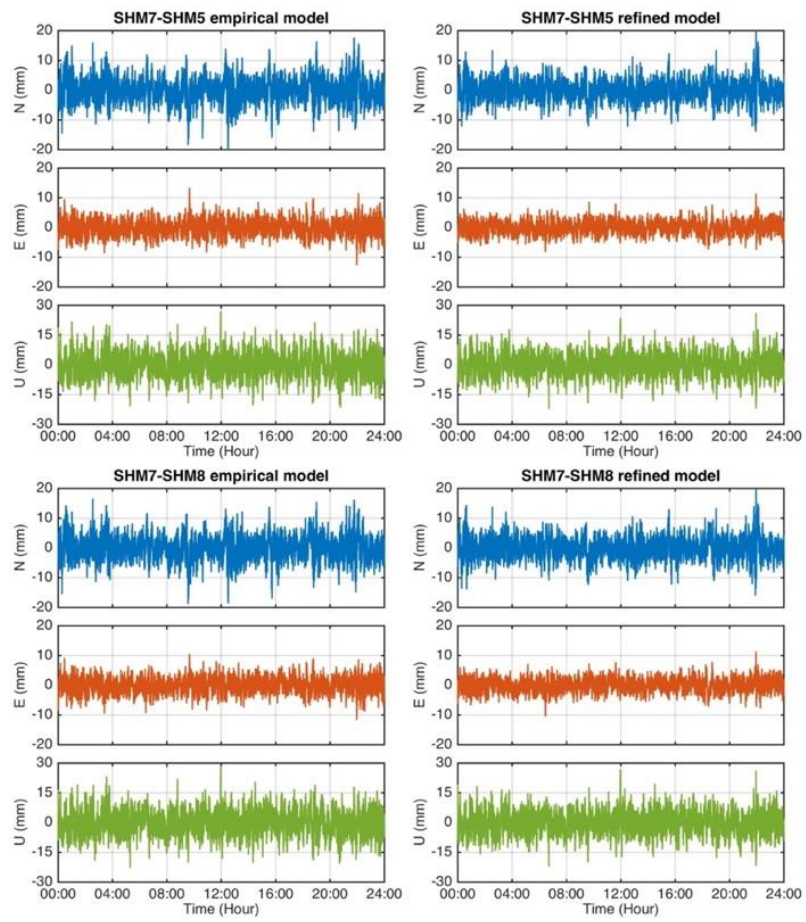

Fig 8 Baseline errors of short-baseline formed by LEICA receiver pair (top) and PANDA pair (bottom) estimated with DD method. In the data processing, we use the empirical model and the refined model respectively as the observation stochastic model.

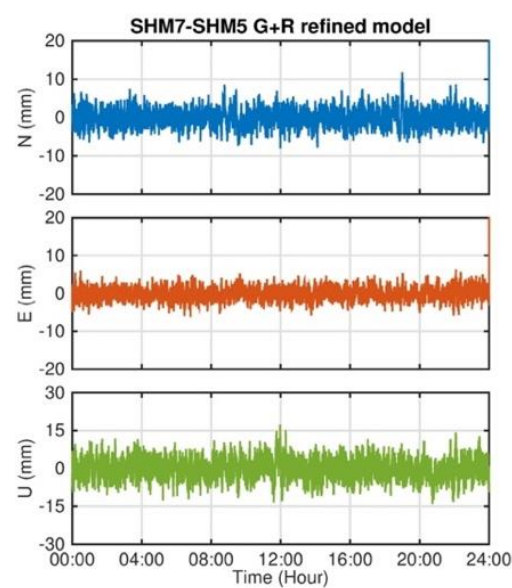

Fig 9 Baseline errors of integrated GPS and GLONASS data processing for SHM7-SHM5 with refined stochastic model. 
Then, we process the integrated GPS and GLONASS data for SHM7-SHM5 with the refined model in Table 3. The STD values for the baselines solutions are also listed in Table 5. Compared with the GPS only empirical model solutions, the precision improves up to $30 \%$ to $40 \%$.

Table 5 STD of short-baseline solutions, and the improvements that the refined model solutions (GPS and integration of GPS and GLONASS) have compared with GPS empirical model.

\begin{tabular}{|c|c|c|c|c|c|c|c|c|c|c|c|c|}
\hline & \multicolumn{3}{|c|}{$\begin{array}{l}\text { G Empirical Model } \\
(\mathrm{mm})\end{array}$} & \multicolumn{3}{|c|}{$\begin{array}{l}\text { G Refined Model } \\
\qquad(\mathrm{mm})\end{array}$} & \multicolumn{3}{|c|}{$\begin{array}{l}\text { G+R Refined Model } \\
\qquad(\mathrm{mm})\end{array}$} & \multicolumn{3}{|c|}{$\begin{array}{l}\text { Improvement (\%) (G Refined Model/ } \\
\text { G+R Refined Model) }\end{array}$} \\
\hline & $\mathrm{N}$ & $\mathrm{E}$ & $\mathrm{U}$ & $\mathrm{N}$ & $\mathrm{E}$ & $\mathrm{U}$ & $\mathrm{N}$ & $\mathrm{E}$ & $\mathrm{U}$ & $\mathrm{N}$ & $\mathrm{E}$ & $\mathrm{U}$ \\
\hline SHM5-SHM7 & 3.5 & 2.2 & 5.2 & 2.9 & 1.8 & 4.7 & 2.0 & 1.5 & 3.5 & $17.1 / 43.9$ & $18.2 / 31.8$ & $9.6 / 32.7$ \\
\hline SHM6-SHM7 & 3.5 & 2.2 & 5.2 & 2.9 & 1.8 & 4.7 & 2.0 & 1.5 & 3.5 & $17.1 / 43.9$ & $18.2 / 31.8$ & $9.6 / 32.7$ \\
\hline SHM8-SHM7 & 3.5 & 2.2 & 5.2 & 2.9 & 1.8 & 4.7 & - & - & - & $17.1 /-$ & $18.2 /-$ & 9.6/- \\
\hline SHM9-SHM7 & 3.5 & 2.2 & 5.2 & 2.9 & 1.8 & 4.7 & - & - & - & $17.1 /-$ & $18.2 /-$ & $9.6 /-$ \\
\hline & & 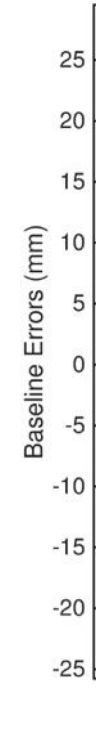 & $\begin{array}{l}\text { 青 } \\
+\end{array}$ & 重 & & $\begin{array}{r}1 \\
1 \\
\frac{1}{1} \\
+ \\
+ \\
+ \\
+\end{array}$ & $\stackrel{1}{\underbrace{}_{1}}$ & 兒 & $\frac{1}{7}$ & 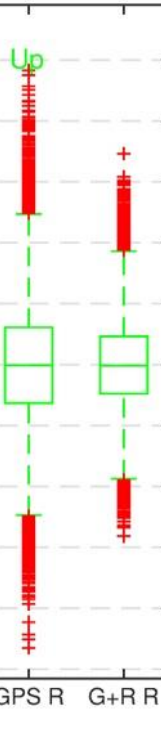 & & \\
\hline
\end{tabular}

Fig 10 Box plots of SHM7-SHM5 baseline errors. (GPS E - GPS single system with empirical stochastic model,

GPS R - GPS single system with refined stochastic model, G+R R - GPS/GLONASS system with refined stochastic model)

Additionally, for better distinction, the box plots of SHM7-SHM5 baseline errors are shown in Fig 10. On each box, the central mark is the median, the edges of the box are the 25th and 75th percentiles, and the whiskers extend to the extreme data points. We can see the extreme data points and the values within the 25th and 75th percentiles are all trend to be smaller after applying refined stochastic model and using multi-GNSS observations.

Fig 11 is presented the spectra of the baseline errors of SHM5-SHM7 in Fig 8 and Fig 9. It is found that the three data processing schemes show the same noise characteristics, with $0.001 \mathrm{~Hz}$ to 
$0.03 \mathrm{~Hz}$ colored noise and white noise in other frequency bands. However, the noise level for GPSonly with the empirical model is the highest in all directions and all frequency bands and the lowest is found in GPS+GLONASS data with the refined stochastic model. Though the noise level reduction is small comparing with the empirical model, the refined stochastic model could be beneficial for the natural frequency detection in the vibration signals.
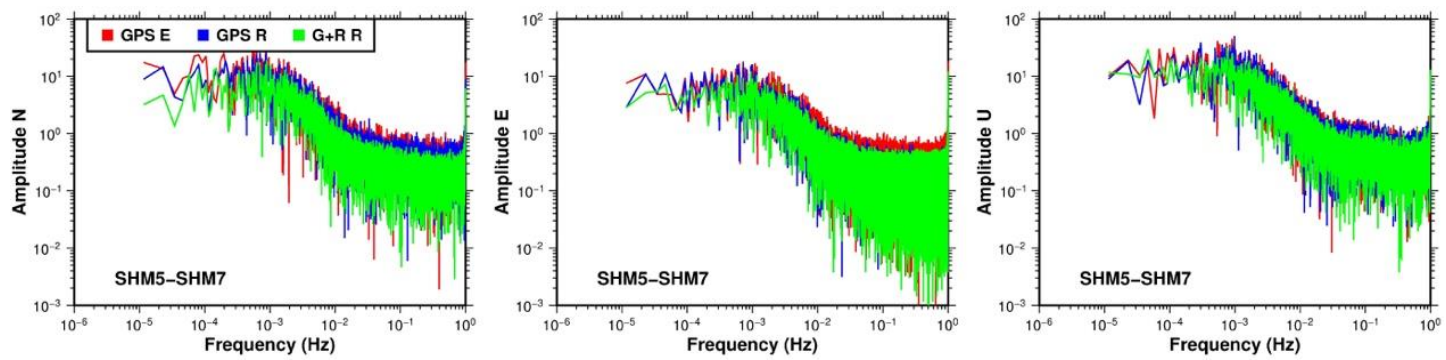

Fig 11 Spectral analysis for the data processing of SHM5-SHM7. (GPS E - GPS single system with empirical stochastic model, GPS R - GPS single system with refined stochastic model, G+R R - GPS/GLONASS system with refined stochastic model).

To comprehensively state the performance of the realistic stochastic model in GNSS data processing, the paper also carried out a single epoch ambiguity resolution test, which means the data of one epoch is resolved independently with no precious epochs' solutions accumulated. Then, the single-epoch ambiguity success rate (ASR) is counted, which is the ratio of epochs with correctly resolved ambiguities with respect to the number of all processed epochs (Paziewski and Wielgosz 2017). The ratio threshold is set to 2 . To reduce the computation burden, the sampling rate of the GPS and GLONASS data is re-sampled to $15 \mathrm{~s}$ with TEQC software in advance (Estey and Meertens 1999). That means 5760 epochs in total a day are available for the test. The results are shown in Fig 12.
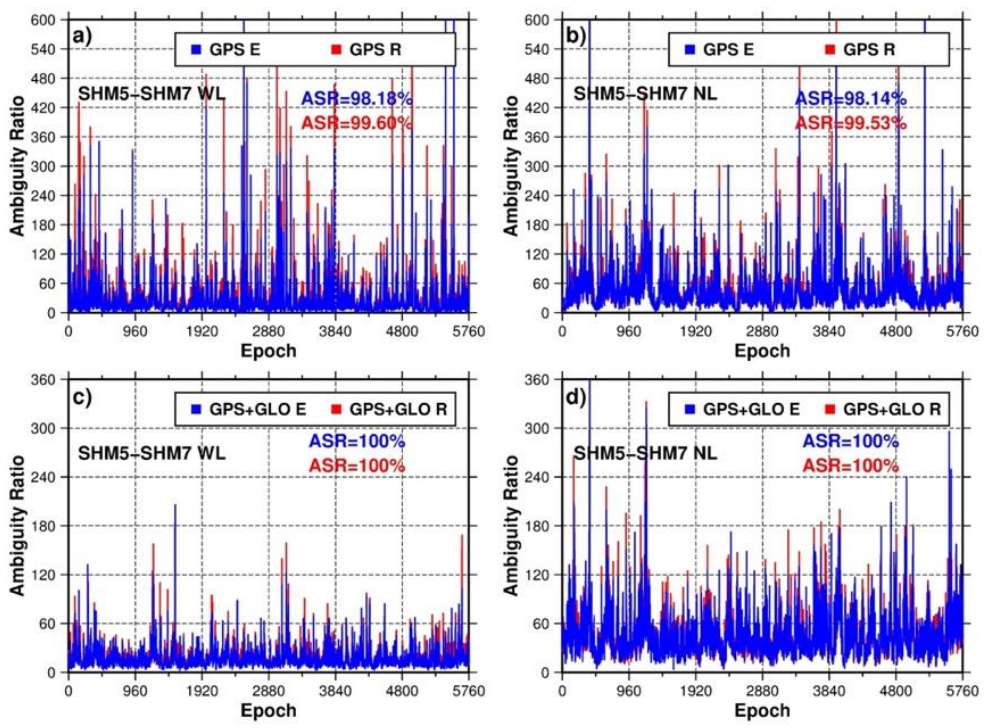
Fig 12 Ratio of single epoch ambiguity resolution of SHM5-SHM7. (GPS E - GPS single system with empirical stochastic model, GPS R - GPS single system with refined stochastic model, G+R R - GPS/GLONASS system with refined stochastic model). The sampling rate of the data has been re-sampled to $15 \mathrm{~s}$ by TEQC software (Estey and Meertens 1999).

One can found that, since the data quality is good enough for this session, the ASR is higher than $98 \%$ for GPS-only data regardless of empirical and refined model. However, the ASR of refined model is slightly better than the empirical model with higher than $99 \%$. After combined with GLONASS data, the ASR can achieve to $100 \%$ for both of the empirical and refined model. From the ratio value of every epoch, however, we can noticed that the refined model is larger than the empirical model most of the time. Therefore, the refined stochastic model may give a more realistic weight for the observations, which will be beneficial for the ambiguity resolution.

\subsection{Performance of the refined stochastic model and multi-GNSS used on the real-life}

\section{bridge monitoring}

In order to show the application performance of the realistic stochastic model and multi-GNSS on the bridge monitoring, we used the refined model estimated previously on the real-life bridge monitoring data in GeoSHM project.

SHM4 is one of the monitoring stations setting on the top of the southern tower of the Forth Road Bridge (FRB). The reference station is on the roof of bridge control room. Both of them equipped with the LEICA GM30 receivers and the LEIAR 10 antennas (same with the zerobaselines and the short-baselines experiments). Sampling rate is set to $20 \mathrm{~Hz}$. Fig 13 depicts the location of SHM4 on the FRB and the defined Bridge Coordinate System (BCS). As we can see, the sky view SHM4 is clear above the elevation angle $10^{\circ}$.

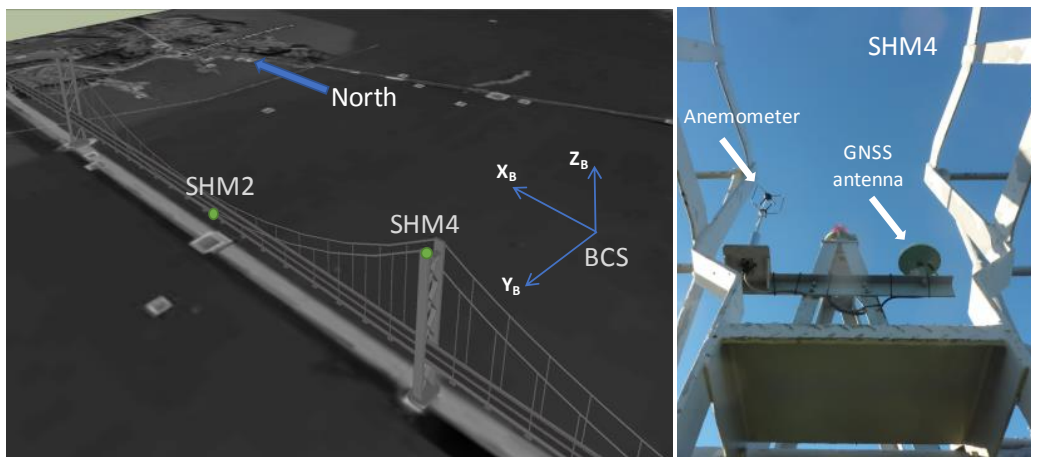

Fig 13 The defined Bridge Coordinate System (BCS) and antenna setting-up at SHM4. 

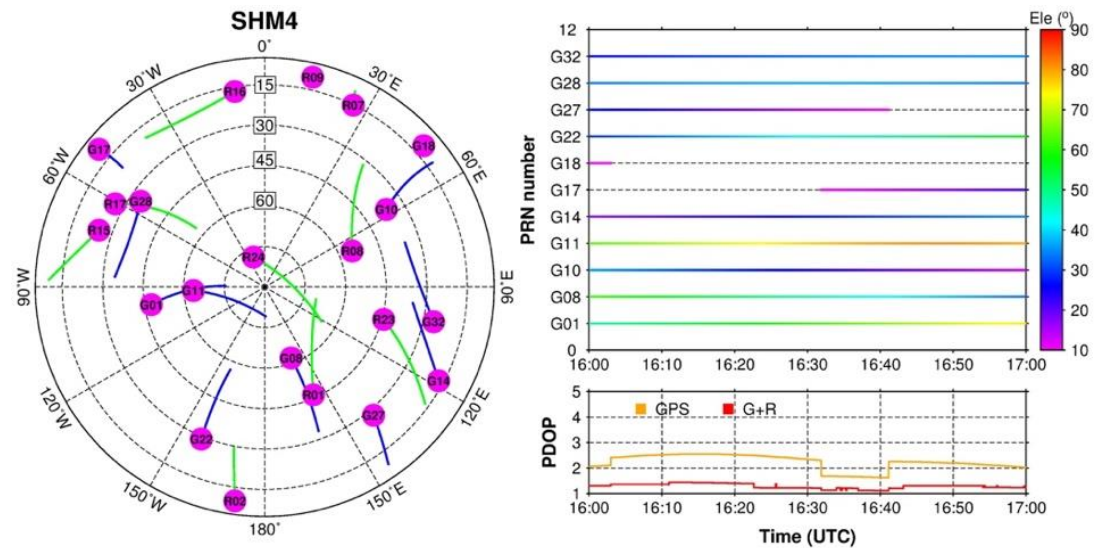

Fig 14 Satellite visible during the data session.

In this study, we selected a specific session during 16:00-17:00 on July $25^{\text {th }} 2017$, when there is an example to demonstrate the application of the realistic stochastic model and multi-GNSS to deal with the false distortion signal. However, this is a common phenomenon that can be seen all the times at all stations. Fig 14 shows the sky plot of SHM4 during this hour. The right panel gives the time lines of the tracked GPS satellites with the elevation variation indicated by color bar, and the PDOP values.

Table 6 Four GNSS data processing schemes applied in the analysis.

\begin{tabular}{llll}
\hline Schemes & Satellite System & Stochastic Model & Cutoff Elevation Angle \\
\hline a & GPS & Model B with $a=b=3 m m$ & 10 \\
b & GPS & Model B with $a=b=3 m m$ & 15 \\
c & GPS & Model A with parameters in Table 3 & 10 \\
d & GPS+GLONASS & Model A with parameters in Table 3 & 10 \\
\hline
\end{tabular}

For comparing purpose, we processed the GNSS data of SHM4 in the four schemes listed in Table 6. Because Model A always gives the better fitting result, in schemes (c) and (d), Model A is applied. Fig 15 denotes the displacement time series with the mean value removed for schemes (a) and (b). The high frequency components of the displacement are also shown, which were obtained by using the eight-order Type 1 Chebyshev high-pass digital filter with pass-band $0.05 \mathrm{~Hz}$ and stopband $1.1 \mathrm{~Hz}$ (Meng et al., 2007, Górski 2017), as natural frequencies of the bridge are commonly shown in this band. 

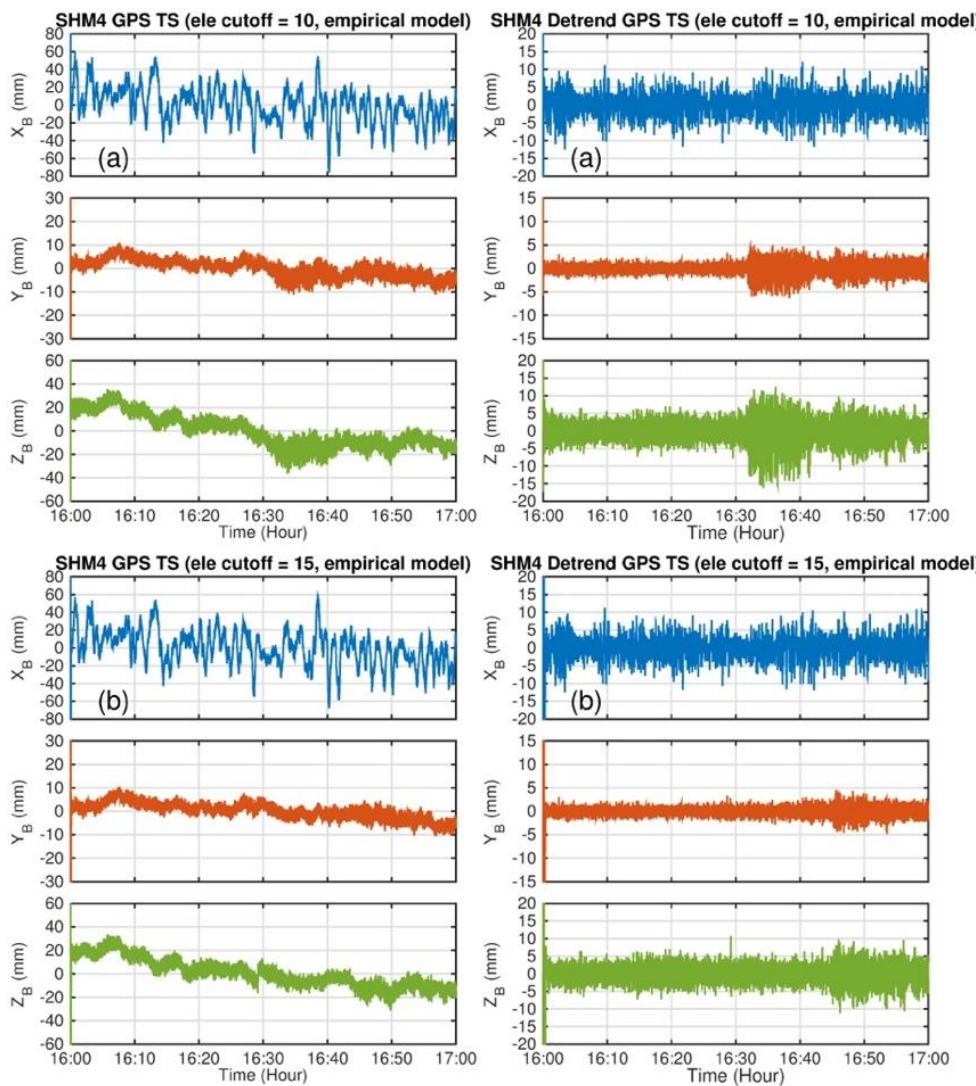

Fig 15 Structural displacements and their high frequency components for empirical model schemes (a) and (b).

For Fig 15(a), the displacements in $X_{B}$ is obvious, which is caused by the traffic load. For $Y_{B}$ and $Z_{B}$ component, the displacements are small and they are coupled together. After bandpass filter processing, the vibration signal in $X_{B}$ is clearly shown. For $Y_{B}$ and $Z_{B}$ component, only noise signal within $2 \mathrm{~mm}$ in $Y_{B}$ and $5 \mathrm{~mm}$ in $Z_{B}$ exist between 16:00 to 16:30. However, it seems to be an excitation at 16:33. The amplitude increases to $5 \mathrm{~mm}$ and $10 \mathrm{~mm}$ in $Y_{B}$ and $Z_{B}$ respectively. After checking with wind and traffic data, we know that the wind strength, direction, and the traffic load had no distinct variations. As seen in Fig 14, around 16:33, the G17 is rising into the tracked satellites list and the elevation is low. Fig 16 depicts the posteriori residuals for GPS L1 observations. We can see that the posteriori residuals of G17 is extremely larger than other satellites. Therefore, we can conclude that the signal between 16:30 to 16:40 are large noise caused by G17.

Note that this may cause two problems. Firstly, the large noise can cover the small amplitude vibration signals. Secondly, it could be recognised as a signal to be extracted from the displacements. In order to reduce the false signal, we rise up the cutoff elevation to $15^{\circ}$ in scheme (b). We can see that the signal between 16:30 and 16:40 is excluded. However, the noise is still large between 16:45 
to 17:00. From Fig 14, it indicates that this is because the dropping of G27. The PDOP value is changing and the geometry strength is becoming weak.

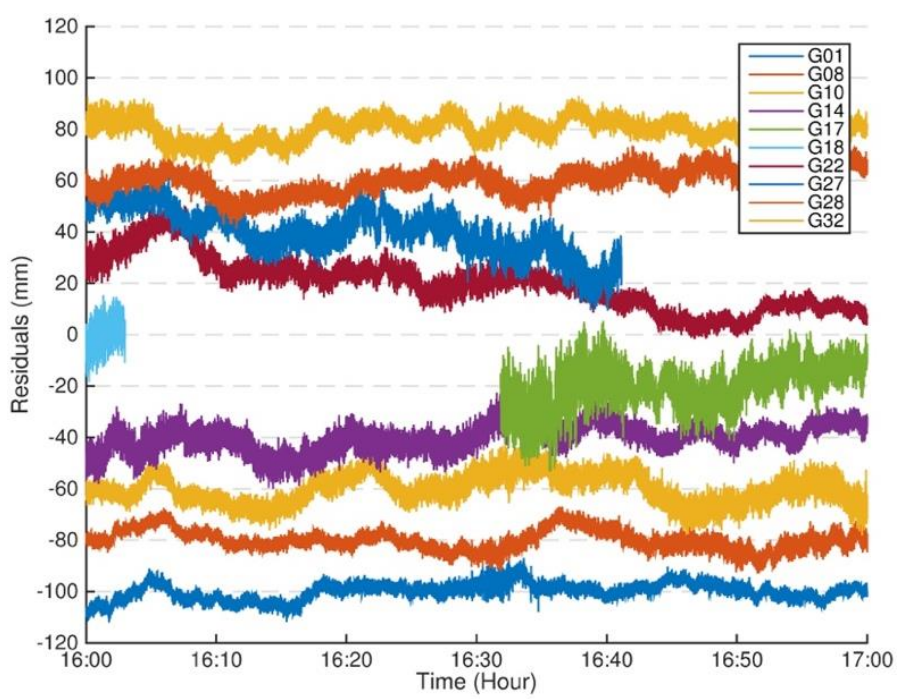

Fig 16 Posteriori residuals for GPS L1 observations. There is an upward shift of $20 \mathrm{~mm}$ successively from G01.

As previously mentioned, the stochastic model describes the observation precisions and weights the observations in coordinate resolution. Joint use of the observations from multi-GNSS can enhance the geometry strength. Thus, we process the data with scheme (c) and (d). The results are shown in Fig 17. Compared with scheme (a), the amplitude of noise in scheme (c) between 16:30 to $16: 40$ reduces to only half the size of scheme (a). However, the large noise is still exist. After processing the data with integrated GPS and GLONASS, we can see that the noise is completely excluded. As seen in Fig 14, the PDOP values during the whole hour is stable. The rising and dropping satellites will not have extreme effects on positioning. More satellites observed can also smooth the large residual errors of low elevation satellite, which makes a low level noise and stable precision for the bridge monitoring time series. 

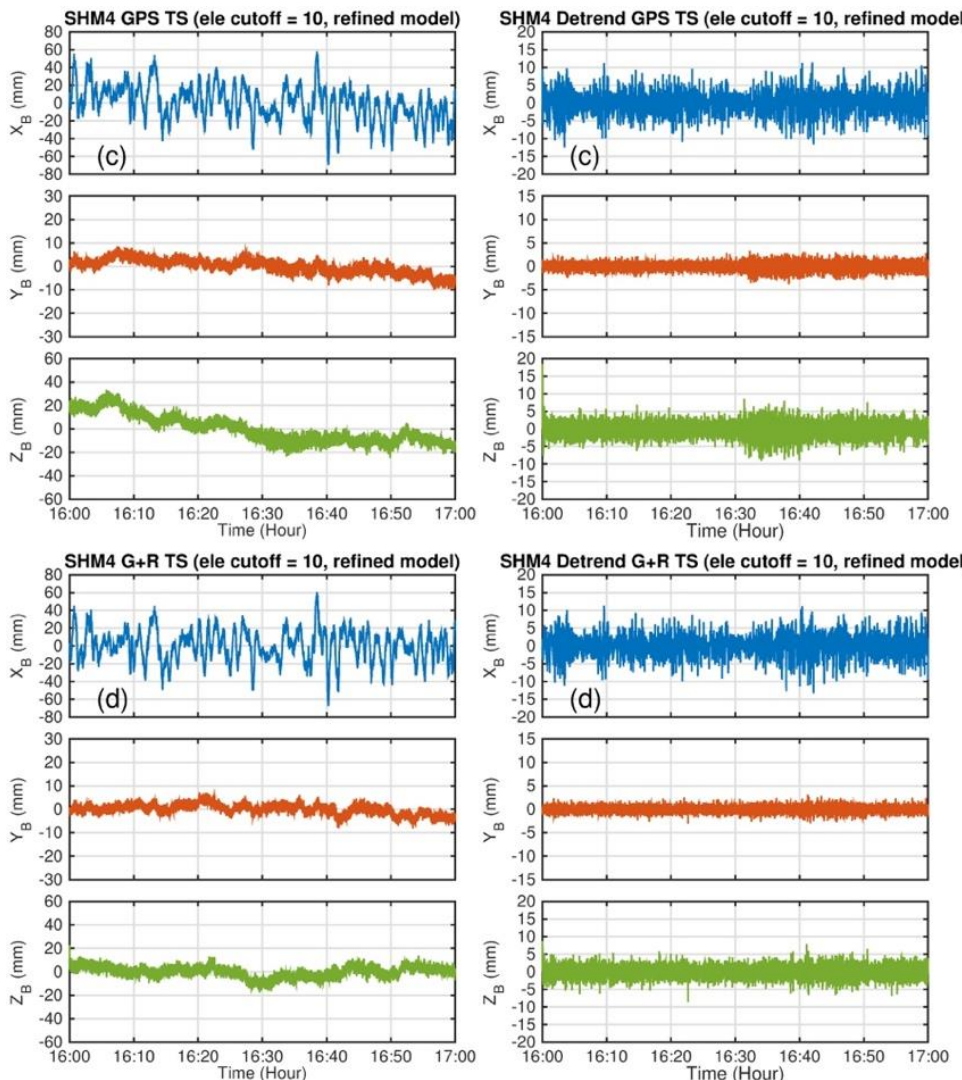

Fig 17 Structural displacements and their high frequency components for refined model schemes (c) and (d).
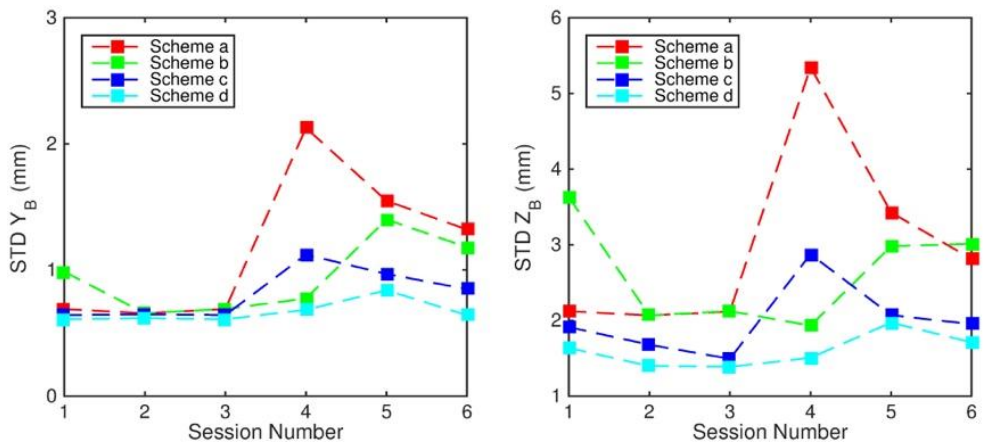

Fig 18 STD calculations for 6 sessions (every 10 minutes) in $Y_{B}$ and $Z_{B}$ directions.

Fig 18 shows the STD values for every 10 minutes in $Y_{B}$ and $Z_{B}$ directions. As shown, even though the STDs of displacements in $0.05 \mathrm{~Hz}-1.1 \mathrm{~Hz}$ are only within several millimeters, the STDs of noise variations are larger than $1 \mathrm{~mm}$ and $3 \mathrm{~mm}$ in $Y_{B}$ and $Z_{B}$ directions for GPS with the empirical stochastic model, which could be recognized as a response signal. The response signals can also be covered by the large noise. Fig 19 shows the spectra of the baseline errors of SHM4-SHM1 for scheme a, c, and d. One can clearly notice that, except for the noise level reduction in all frequency bands for the refined model, the amplitude of natural frequency of $0.18 \mathrm{~Hz}$ are significantly reduced in scheme d. However, the spectral peak at $0.18 \mathrm{~Hz}$ is still noticed. It demonstrates that the noise 
amplifies the amplitude of the natural frequency of the bridge, which will provide a wrong parameter for the bridge statement assessment.
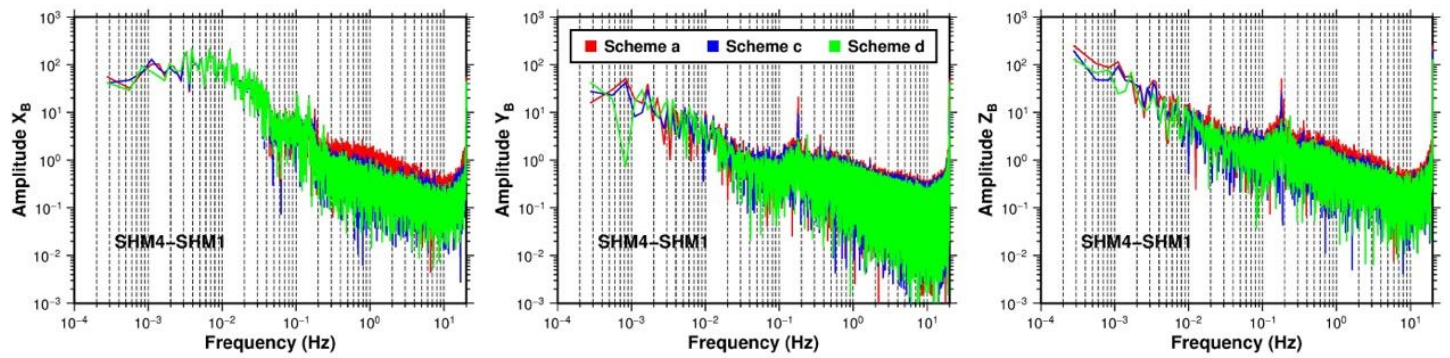

Fig 19 Spectral analysis for the data processing of SHM4-SHM1.
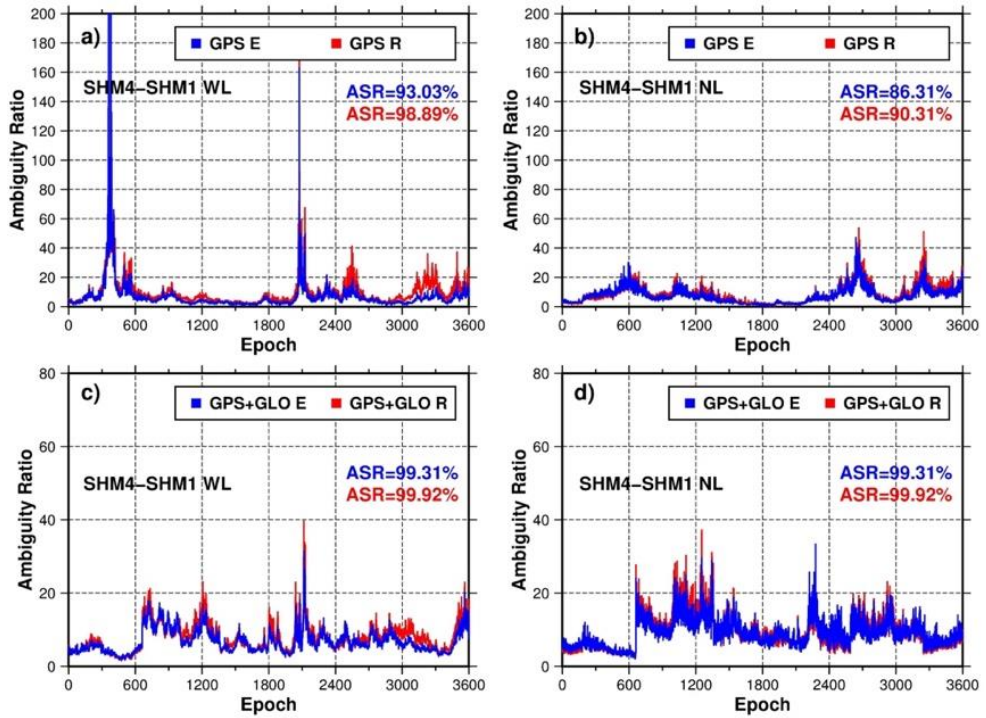

Fig 20 Ratio of single epoch ambiguity resolution of SHM4-SHM1. (GPS E - GPS single system with empirical stochastic model, GPS R - GPS single system with refined stochastic model, G+R R - GPS/GLONASS system with refined stochastic model). The sampling rate of the data has been re-sampled to 1s by TEQC software (Estey and Meertens 1999).

In addition, Fig 20 shows the single epoch ambiguity resolution performance with the data of SHM4-SHM1. Before the experiment, the data were processed to $1 \mathrm{~s}$ by TEQC software as well. From Fig 20, the same phenomenon found in Fig 12 is shown. With the realistic model, the random errors in the GPS/GLONASS measurements can be reasonably described to improve the GPS/GLONASS integer ambiguity resolution success rate (Amiri-Simkooei et al. 2016).

\section{Conclusion}

In this study, we first proposed a GPS and GLONASS carrier phase elevation-dependent precision modelling method. Then, based on a testing platform served for the GeoSHM project with a group of zero-baselines and short-baselines, we analyse the impacts of receiver internal noise and refined GNSS stochastic model on the positioning. At last, the contribution of integration of GPS 
and GLONASS is also introduced. After the experimental analysis, some useful conclusions can be summarised as follows:

- The precision estimated for GPS and GLONASS measurements is overall elevation-dependent for both zero-baseline and short-baseline. In the zero-baseline experiment, the internal noise features are accounting for the systems, receiver brands and frequencies. For GPS observations, the precision of L1 is better than L2. The cost effective receiver PANDA has a high level internal noise than LEICA. For GLONASS, the elevation-dependent characteristic is not obvious compared with GPS. The precision of GLONASS L2 observation is slightly better than L1.

- In the short-baseline experiment, however, we got almost the same precisions for GPS observations in L1 and L2 frequencies. The PANDA receivers with high internal noise can have the same performance with the LEICA ones. For GLONASS, the precision of L2 is worse than L1, and they show a lower mean precision compared with GPS. However, at low elevation region, they show a little bit higher precision than GPS. After fitting observation precisions of GPS and GLONASS by elevation-dependent functions, we use them in the DD data processing, and compare with the empirical model solutions. We found that the refined model can improve the precision of the baseline time series from $10 \%$ to $20 \%$. If GLONASS data were joint, the precision can further improve by $30 \%$ to $40 \%$. From further analysis with box plots, we found that the extreme data points and the values within the 25th and 75th percentiles are all trend to be smaller after applying the refined stochastic model and using multi-GNSS observations.

- By using the refined model estimated with the short-baseline data into the real-life bridge monitoring data processing, the large noise caused by the observations of the low elevation satellites and rising and dropping satellites can be reduced. The integration of GPS and GLONASS with refined model tend to completely eliminate the false distortion signals. The ambiguity resolution rate is also improved with the refined stochastic model, since the random errors in the GPS/GLONASS measurements are reasonably described.

Finally, the paper suggests that, for the long-term bridge deformation monitoring, the receiver internal noise, the realistic stochastic model can be evaluated and estimated in advance. Multi-GNSS data could be applied to achieve high precision of displacements and reliable vibration parameters. 


\section{Acknowledgment}

This work is supported by "the Open Foundation of Key Laboratory of Precise Engineering and Industry Surveying of National Administration of Surveying, Mapping and Geoinformation" (Grant No. PF2017-8). This research was also supported by the National Natural Science Foundation of China (Grant Nos. 41525014, 41304007 and 41210006), the Program for Changjiang Scholars of the Ministry of Education of China. The Chinese Scholarship Council (CSC) has provided the first author a scholarship which allows him to visit the University of Nottingham for two years to research and study in the UK from November 2016. ESA is acknowledged for sponsoring GeoSHM feasibility an Demo project.

\section{References}

[1] Amiri-Simkooei, A. R. Application of least squares variance component estimation to errors-invariables models. Journal of Geodesy. 2013a, 87(10-12), 935-944.

[2] Amiri-Simkooei, A. R. Noise in multivariate GPS position time-series. Journal of Geodesy. 2009, 83(2), 175-187.

[3] Amiri-Simkooei, A. R.; Jazaeri, S.; Zangeneh-Nejad, F.; Asgari, J. Role of stochastic model on GPS integer ambiguity resolution success rate. GPS solutions. 2016, 20(1), 51-61.

[4] Amiri-Simkooei, A. R.; Tiberius, C. C. J. M. Assessing receiver noise using GPS short baseline time series. GPS solutions. 2007, 11(1), 21-35.

[5] Amiri-Simkooei, A. R.; Zangeneh-Nejad, F.; Asgari, J. Least-squares variance component estimation applied to GPS geometry-based observation model. Journal of Surveying Engineering. 2013b, 139(4), 176-187.

[6] Chan, W. S.; Xu, Y. L.; Ding, X. L.; Dai, W. J. An integrated GPS-accelerometer data processing technique for structural deformation monitoring. Journal of Geodesy. 2006, 80(12), 705-719.

[7] Chu, F. Y.; Yang, M. BeiDou System (BDS) Triple-Frequency Ambiguity Resolution without Code Measurements. Remote Sensing. 2018, 10(5), 675.

[8] Chu, F. Y.; Yang, M.; Wu, J. A new approach to modernized GPS phase-only ambiguity resolution over long baselines. Journal of Geodesy. 2016, 90(3), 241-254.

[9] Dach, R.; Hugentobler, U.; Fridez, P.; Meindl, M. Bernese GPS software version 5.0. Astronomical Institute, University of Bern, 2007, 640, 114.

[10] DAI, W.; Ding, X. Single Epoch Ambiguity Resolution in Structure Monitoring Using GPS. 
Geomatics and Information Science of WuhanUniversity. 2007, 32(3), 234.

[11] Elnabwy M. T.; Kaloop M. R.; Elbeltagi E. Talkha steel highway bridge monitoring and movement identification using RTK-GPS technique. Measurement. 2013, 46(10), 4282-4292.

[12] Estey L H, Meertens C M. TEQC: the multi-purpose toolkit for GPS/GLONASS data[J]. GPS solutions, 1999, 3(1): 42-49.

[13] Geng, J.; Shi, C. Rapid initialization of real-time PPP by resolving undifferenced GPS and GLONASS ambiguities simultaneously. Journal of Geodesy. 2017, 91(4), 361-374.

[14] Górski P. Dynamic characteristic of tall industrial chimney estimated from GPS measurement and frequency domain decomposition. Engineering Structures. 2017, 148, 277-292.

[15] Guo, J.; Xu, L.; Dai, L.; McDonald, M.; Wu, J.; Li, Y. Application of the real-time kinematic global positioning system in bridge safety monitoring. Journal of Bridge Engineering. 2005, 10(2), 163 168.

[16] Han, H.; Wang, J.; Meng, X.; Liu, H. Analysis of the dynamic response of a long span bridge using GPS/accelerometer/anemometer under typhoon loading. Engineering Structures. 2016, 122, 238 250.

[17] Herring, T. A.; King, R. W.; McClusky, S. C. Gamit reference manual: GPS Analysis at MIT, Release 10.4. Department of Earth, Atmospheric, and Planetary Sciences. 2010.

[18] Jiang, W.; Xi, R.; Chen, H.; Xiao, Y. Accuracy analysis of continuous deformation monitoring using BeiDou Navigation Satellite System at middle and high latitudes in China. Advances in Space Research. 2017, 59(3), 843-857.

[19] Lagler K, Schindelegger M, Böhm J, et al. GPT2: Empirical slant delay model for radio space geodetic techniques[J]. Geophysical research letters, 2013, 40(6): 1069-1073.

[20] Li P.; Zhang, X. Integrating GPS and GLONASS to accelerate convergence and initialization times of precise point positioning. GPS solutions. 2014, 18(3), 461-471.

[21] Li, B. F.; Shen, Y. Z.; Xu, P. L. Assessment of stochastic models for GPS measurements with different types of receivers. Chinese Science Bulletin. 2008, 53(20), 3219-3225.

[22] Li, B. Stochastic modeling of triple-frequency BeiDou signals: estimation, assessment and impact analysis. Journal of Geodesy. 2016, 90(7), 593-610.

[23] Li, B.; Feng, Y.; Gao, W.; Li, Z. Real-time kinematic positioning over long baselines using triplefrequency BeiDou signals. IEEE Transactions on Aerospace and Electronic Systems. 2015b, 51(4), 
3254-3269.

[24] Li, B.; Lou, L.; Shen, Y. GNSS elevation-dependent stochastic modeling and its impacts on the statistic testing. Journal of Surveying Engineering. 2015a, 142(2), 04015012.

[25] Li, B.; Zhang, L.; Verhagen, S. Impacts of BeiDou stochastic model on reliability: overall test, wtest and minimal detectable bias. GPS Solutions. 2017a, 21(3),1095-1112.

[26] Li, P.; Zhang, X.; Guo, F. Ambiguity resolved precise point positioning with GPS and BeiDou. Journal of Geodesy. 2017b, 91(1), 25-40.

[27] Liu, Y.; Ye, S.; Song, W.; Lou, Y.; Gu, S. Rapid PPP ambiguity resolution using GPS+ GLONASS observations. Journal of Geodesy. 2017, 91(4), 441-455.

[28] Liu, Z.; He, X.; Zhang, S.; Wang, J. Dynamic triple-difference method for single frequency GPS deformation monitoring. Journal of Tongji University. Natural Science. 2011, 39(7), 1074-1078.

[29] Meng, X.; Dodson, A. H.; Roberts, G. W. Detecting bridge dynamics with GPS and triaxial accelerometers. Engineering Structures. 2007, 29(11), 3178-3184.

[30] Meng, X.; Nguyen, D. T.; Xie, Y.; Owen, J. S.; Psimoulis, P.; Ince, S.; Chen, Q.; Ye, J.; Bhatia, P. Design and implementation of a new system for large bridge monitoring-GeoSHM. Sensors. $2018,18(3), 775$.

[31] Meng, X.; Roberts, G. W.; Cosser, E.; Dodson, A. H.; Barnes, J.; Rizos, C. Real-time bridge deflection and vibration monitoring using an integrated GPS/accelerometer/pseudolite system. In Proc of 11th International Symposium on Deformation Measurements, International Federation Surveyors (FIG), Commission 6-Engineering Surveys, Working Group. 2003, pp. 25-28.

[32] Meng, X.; Roberts, G. W.; Dodson, A. H.; Cosser, E.; Barnes, J.; Rizos, C. Impact of GPS satellite and pseudolite geometry on structural deformation monitoring: analytical and empirical studies. Journal of Geodesy. 2004, 77(12), 809-822.

[33] Moschas, F.; Stiros, S. Measurement of the dynamic displacements and of the modal frequencies of a short-span pedestrian bridge using GPS and an accelerometer. Engineering Structures. 2011, $33(1), 10-17$

[34] Odolinski, R.; Teunissen, P. J.; Odijk, D. Combined BDS, GALILEO, QZSS and GPS singlefrequency RTK. GPS solutions. 2015, 19(1), 151-163.

[35] Paziewski J, Wielgosz P. Investigation of some selected strategies for multi-GNSS instantaneous RTK positioning[J]. Advances in Space Research, 2017, 59(1): 12-23. 
[36] Peppa I, Psimoulis P, Meng X. Using the signal - to - noise ratio of GPS records to detect motion of structures. Structural Control and Health Monitoring, 2018, 25(2): e2080.

[37] Psimoulis, P.; Pytharouli, S.; Karambalis, D.; Stiros, S. Potential of Global Positioning System (GPS) to measure frequencies of oscillations of engineering structures. Journal of Sound and Vibration. 2008, 318(3), 606-623.

[38] Qian, K.; Wang, J.; Hu, B. A posteriori estimation of stochastic model for multi-sensor integrated inertial kinematic positioning and navigation on basis of variance component estimation. The Journal of Global Positioning Systems. 2016, 14(1), 5.

[39] Roberts, G. W.; Tang, X. The use of PSD analysis on BeiDou and GPS $10 \mathrm{~Hz}$ dynamic data for change detection. Advances in Space Research. 2017, 59(11), 2794-2808.

[40] Schön, S.; Brunner, F. K. A proposal for modelling physical correlations of GPS phase observations. Journal of Geodesy. 2008, 82(10), 601-612.

[41] Tamura, Y.; Matsui, M.; Pagnini, L. C.; Ishibashi, R.; Yoshida, A. Measurement of wind-induced response of buildings using RTK-GPS. Journal of Wind Engineering and Industrial Aerodynamics. 2002, 90(12-15), 1783-1793.

[42] Teunissen, P. J. G. Influence of ambiguity precision on the success rate of GNSS integer ambiguity bootstrapping. Journal of Geodesy. 2007, 81(5), 351-358.

[43] Teunissen, P. J. G.; Odolinski, R.; Odijk, D. Instantaneous BeiDou+ GPS RTK positioning with high cut-off elevation angles. Journal of geodesy. 2014, 88(4), 335-350.

[44] Wanninger, L. Carrier-phase inter-frequency bias of GLONASS receivers. J Geod. 2012, 86(2), $139-148$.

[45] Xiong, C.; Lu, H.; Zhu, J. Operational Modal Analysis of Bridge Structures with Data from GNSS/Accelerometer Measurements. Sensors. 2017, 17(3), 436.

[46] Yang, L.; Li, B.; Li, H.; Rizos, C.; Shen, Y. The influence of improper stochastic modeling of Beidou pseudoranges on system reliability. Advances in Space Research. 2017, 60(12), 2680-2690.

[47] Yi, T. H.; Li, H. N.; Gu, M. Experimental assessment of high-rate GPS receivers for deformation monitoring of bridge. Measurement. 2013, 46(1), 420-432.

[48] Yu, J.; Meng, X.; Shao, X.; Yan, B.; Yang, L. Identification of dynamic displacements and modal frequencies of a medium-span suspension bridge using multimode GNSS processing. Engineering Structures. 2014, 81, 432-443. 
[49] Yu, J.; Yan, B.; Meng, X.; Shao, X.; Ye, H. Measurement of bridge dynamic responses using network-based real-time kinematic GNSS technique. Journal of Surveying Engineering. 2016, 142(3), 04015013.

[50] Yu, W.; Ding, X.; Dai, W.; Chen, W. Systematic error mitigation in multi-GNSS positioning based on semiparametric estimation. Journal of Geodesy. 2017, 91(12), 1491-1502. 